\title{
Diversidad genérica de hormigas (Himenópteros: Formicidae) en ambientes de bosque, borde de bosque y áreas cultivadas tres Comunidades del Municipio de Coripata, Nor Yungas \\ Departamento de La Paz, Bolivia
}

\section{Generic diversity of ants (Himenopteros: Formicidae) in forest set, forest border and areas cultivated three Communities of the Municipality of Coripata, Nor Yungas Department of La \\ Paz, Bolivia}

Mamani-Mamani Beatriz ${ }^{1}$, Loza-Murguia Manuel ${ }^{1,2}$, Smeltekop Hugh $^{1}{ }^{*}$, Almanza Juan Carlos ${ }^{1}$, Limachi Miguel ${ }^{3}$

\begin{tabular}{|c|c|}
\hline Datos del Artículo & Resumen \\
\hline 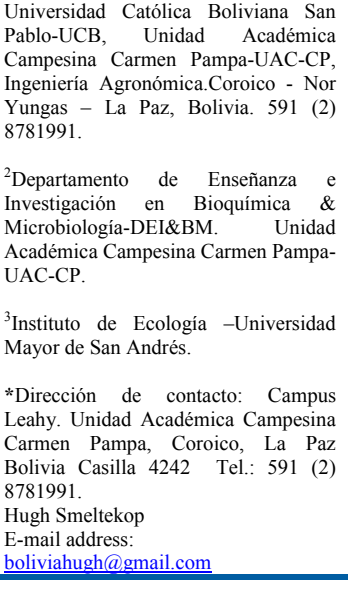 & \multirow[t]{2}{*}{$\begin{array}{l}\text { La biodiversidad es la variedad de todos los vegetales, animales y los microorganismos que llegan a coexistir e } \\
\text { interactuar dentro de un ecosistema para interrelacionarse entre sí. Las hormigas representan la mayor abundancia } \\
\text { dentro de los insectos. La familia Formicidae se caracteriza por la importancia que tiene en los ecosistemas naturales } \\
\text { y por la variedad de funciones ecológicas que cumplen, debido a la asociación con muchas plantas y animales. Tienen } \\
\text { una gran variabilidad en la alimentación y utilizan diversas formas de nidificación; sin embargo son muy escasos los } \\
\text { estudios realizados. La presente investigación se realizó en el municipio de Coripata, donde la familia Formicidae } \\
\text { mostró mayor abundancia y riqueza entre hábitats. Para eso se instalaron las } 5 \text { trampas de caída por sitio (Altuspata, } \\
\text { Choro y Choro Alto) en tres tipos de hábitats diferentes (bosque, borde de bosque y cultivo) durante } 12 \text { meses. Se } \\
\text { encontraron } 15026 \text { individuos distribuidos en } 6 \text { subfamilias con } 26 \text { géneros y } 46 \text { especies y/o morfoespecies } \\
\text { encontradas en total. Esto muestra que existe una gran riqueza y abundancia de hormigas en este ecosistema. La } \\
\text { subfamilia Ecitoninae mostró un mayor número de individuos por la forma de hábito que tienen de ser depredadores y } \\
\text { nómadas. La mayor riqueza y abundancia de hormigas se identificó en el área de bosque del sitio Altuspata. Dentro de } \\
\text { la subfamilia Ecitoninae las especies Labidus spininoides y Labidus praedator presentaron mayor número, y con } \\
\text { respecto a los sitios se tiene similitud en cuanto a la cantidad de individuos. } \\
\text { C 2012. Journal of the Selva Andina Research Society. Bolivia. Todos los derechos reservados. }\end{array}$} \\
\hline \multirow{2}{*}{$\begin{array}{l}\text { Palabras clave: } \\
\text { Formicidae (Hormigas), } \\
\text { biodiversidad, } \\
\text { perturbaciones, } \\
\text { trampas de caída, } \\
\text { Altuspata, Choro y Choro Alto. }\end{array}$} & \\
\hline & Abstract \\
\hline $\begin{array}{l}\text { J Selva Andina Res } \\
\text { Soc. } 2012 ; 3(1): 26-43 .\end{array}$ & $\begin{array}{l}\text { The biodiversity is the variety of all the vegetables, animals and the microorganisms that end up coexisting and } \\
\text { interaction inside an ecosystem to be interrelated to each other. The ants represent the biggest abundance inside the } \\
\text { insects. The family Formicidae is characterized by the importance that has in the natural ecosystems and for the } \\
\text { variety of ecological functions that you/they complete, due to the association with many plants and animals. They } \\
\text { have a great variability in the feeding and they use diverse nidificación forms; however they are very scarce the } \\
\text { carried out studies. The present investigation was carried out in the municipality of Coripata, where the family }\end{array}$ \\
\hline
\end{tabular}




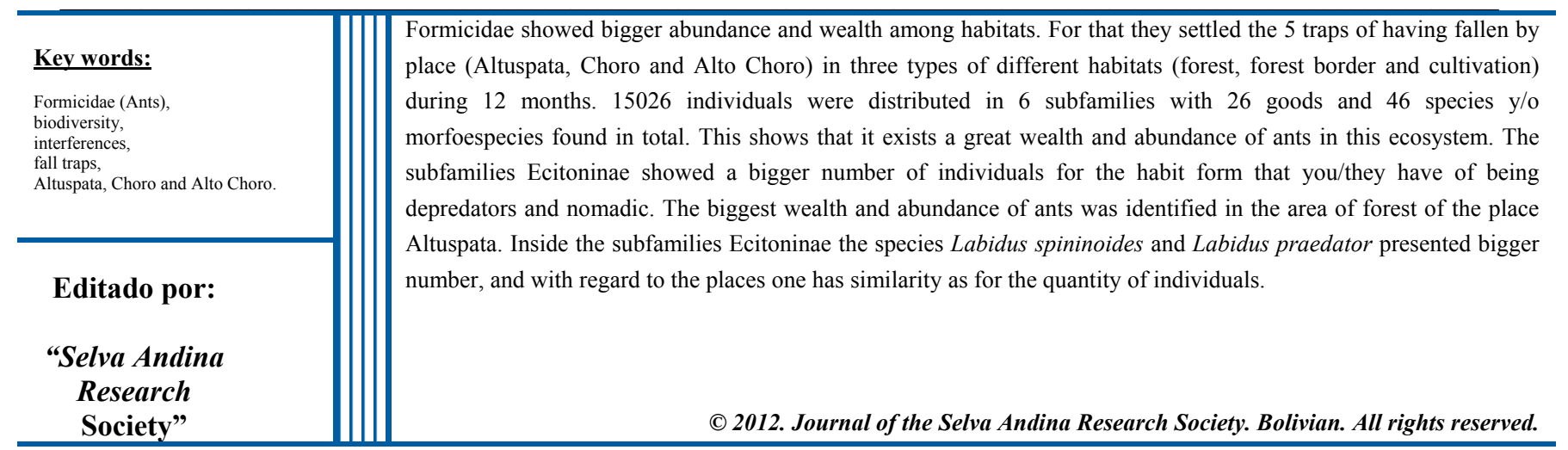

\section{Introducción}

Los insectos se han adaptado a cambios en el medio ambiente, la sobrevivencia y capacidad de adaptación por millones de años Vargas Rojas (1995). Uno de los factores que limita la distribución geográfica de los insectos es su hábito alimenticio, algunos se alimentan de plantas verdes o madera muerta, otros son parásitos de plantas y animales.

LaSalle \& Gauld (1993) indicaron a nivel mundial existen más de 115,000 especies descritas de Hymenoptera. Por su parte, Gauld \& Bolton (1988) estimaron la existencia de al menos 250.000 especies mientras que LaSalle (1993) señaló la probabilidad de que al menos el $75 \%$ de los himenópteros parasitoides no ha sido descrito y que se sabe aún menos de su biología.

Lattke (2003b) expresa que la distribución de las hormigas en la zona neotropical no es uniforme en especies, géneros, algunos géneros de distribución cosmopolita sufrieron radiación en el neotrópico. La abundancia en mirmecofauna a pesar de la escasa información disponible de las diferentes zonas del neotrópico. La región andina representa por Chile, Bolivia y Perú y la neotropical desde el 27 norte de México hasta el centro de Argentina, englobando países como: México, Cuba, Haití, Colombia en la cuenca de Orinoco-Amazonas y Brasil.

Las hormigas presentan una organización completa, con estructura definida, cada casta realiza trabajo específico en la colonia, son altamente sociales y tienen disciplina militar. Se diferencian castas: exploradoras, cortadoras, cargadoras, escoteras, jardineras, soldados, machos alados, hembras aladas y reina, pudiendo variar según la especie (Vergara Castrillón 2005, Fernandéz 2003a).

Las hormigas son uno de los grupos de insectos con mayor diversidad específica y ecológica en las latitudes tropicales, cumplen funciones importantes en todos los ecosistemas y constituyen alrededor del $15 \%$ de la biomasa animal total (Villareal et al 2006), presentan especialización en sus hábitos alimenticios y estrecha relación con especies vegetales en especial de las familias Caesalpinaceae, Fabaceae, Melastomataceae, Cecropiaceae y Rubiaceae, algunas especies se 
alimentan solo de huevos de artrópodos o de otras hormigas (Holldobler \& Wilson 1990).

La familia Formicidae está representada actualmente en la región neotropical por 15 subfamilias: Agroecomyrmecinae, Amblyoponinae, Cerapachyinae, Dolichoderinae, Ecitoninae, Ectatomminae, Formicinae, Heteroponerinae, Leptanilloidinae, Myrmicinae, Paraponerinae, Ponerinae, Proceratiinae, Pseudomyrmecinae y la recientemente descrita Martialinae, que fue descubierta por Rabeling et al (2008) en el Amazonas Brasileño. Fernandez \& Sendoya (2004), Fernandez \& Sharkey (2006), afirman que hay algo más de 11.500 especies de hormigas descritas en 21 subfamilias en el neotrópico y registradas unas 3.100 especies y 120 géneros.

Debido a su posición geográfica y gran diversidad de ecosistemas, la región de Bolivia, en el departamento de La Paz ha sido poco estudiada y merecido escaso interés por parte de los investigadores, sin embargo, de manera específica para este departamento no existen investigaciones publicadas acerca mirmecofauna. Por tal razón, el objetivo de este trabajo determinar la diversidad genérica de Himenópteros de algunas de las subfamilias y géneros de hormigas que están presentes en tres Comunidades del Municipio de Coripata, con el propósito de valorar su importancia y generar información sobre insectos permite planificar el manejo de la producción agrícola.

\section{Materiales y métodos}

Los ensayos se realizaron en las comunidades de
Choro Alto, Choro y Altuspata, perteneciente al municipio de Coripata, segunda sección de la provincia Nor Yungas del departamento de La Paz, a una altitud comprendida entre 1060 a 4000 msnm y temperatura variable entre $11-26{ }^{\circ} \mathrm{C}$. (Gobierno Municipal de Coripata 2006).

Se diseñaron trampas de caída (pitfall) de plástico color rojo de $500 \mathrm{~mL}$, las trampas Malaise, se diseñaron de plástico, pintando su interior de amarillo. Se ubicó en áreas de cultivo de cítricos, hortalizas y otros cultivos de la zona, luego en el borde de bosque y bosque, respectivamente.

Las trampas de caída fueron instaladas en tres localidades diferentes, en baterías de quince recipientes de plástico de $14 \times 11 \mathrm{~cm}$., en círculos haciendo un diámetro de $5 \mathrm{~m}$ colocadas en forma equidistante una de otra, colocándose en el centro de cada anillo la trampa Malaise. Fueron rellenadas de agua a las que se añadía etilenglicol para reducir en lo posible la evaporación del líquido contenido en ellas.

Todas estas trampas estuvieron instaladas un ciclo anual completo; desde de julio de 2008 hasta el junio de 2009, procediéndose a la recogida de sus capturas con una periodicidad de 14 días. El material capturado en las seis trampas "Pitfalls" que formaban cada batería de trampeo se reunía para constituir una única muestra que era conservada en solución $45 \mathrm{~mL}$ de agua y $10 \mathrm{~mL}$ de alcohol al 70\% hasta su clasificación y determinación.

Los especímenes fueron llevados al laboratorio de la sección de invertebrados de la Colección Boliviana de Fauna (CBF) perteneciente al 
Instituto de Ecología de la Universidad Mayor de San Andrés, ciudad de La Paz efectuándose su identificación, nivel subfamilia con claves Bolton et al (1990), Bolton (1994), Palacio \& Fernández (2003). A nivel género se utilizó Lattke (2004), Fernández et al (1996), Palacio \& Fernández (2003), Serna \& Vergara et al (2001), Baroni Urbani (1983). A nivel especie y subespecie se utilizaron claves y parámetros de Watkins (1976), Fernández (2003b), Brown (1963), Lattke (2003a), Sarmiento (2003), Fernández \& Sendoya (2004).

Análisis estadístico. El análisis estadístico de la investigación se realizó con el programa PRIMER 6. Para el índice de Similaridad de (Clarke \& Warwick 2001).

Se utilizo análisis de clúster para representar gráficos de las agrupaciones. Se realizo análisis de escala multidimensional donde los resultados muestran en grupos de similaridad, el SIMPER, muestra la contribución de especies bajo criterios de similitud y disimilitud.

Como último se estimo el número de especies en el ecosistema con modelos matemáticos basados en la acumulación de especies observadas.

La abundancia relativa fue determinada a través de la siguiente fórmula.

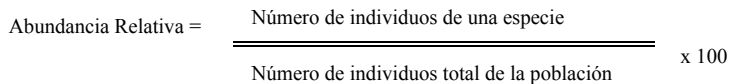

\section{Resultados}

Se capturaron 15026 individuos, se identificaron 45 morfoespecies pertenecientes a 26 géneros distribuidas en 6 subfamilias y 7 tribus, fueron capturadas en la trampa de caída (pitfall) en las tres comunidades en un periodo de 12 meses de estudio, de julio de 2008 hasta el junio de 2009.

Tabla 1 Captura por comunidad de adultos de la familia Formicidae de julio de 2008 a junio de 2009 en los tres hábitats de las comunidades Altuspata, Alto Choro, y Choro municipio de Coripata segunda sección de la provincia Nor Yungas del Departamento de La Paz, Bolivia

\begin{tabular}{|c|c|c|c|c|}
\hline \multirow{3}{*}{ Comunidad } & \multicolumn{4}{|c|}{ Número de Individuos } \\
\hline & \multicolumn{4}{|c|}{ hábitats } \\
\hline & Bosque & $\begin{array}{l}\text { Borde de } \\
\text { Bosque }\end{array}$ & $\begin{array}{l}\text { Área de } \\
\text { Cultivo }\end{array}$ & Total \\
\hline Altuspata & 1933 & 909 & 1828 & 4670 \\
\hline Choro Alto & 881 & 2129 & 1219 & 4229 \\
\hline Choro & 682 & 4541 & 904 & 6127 \\
\hline Total & 3496 & 7579 & 3951 & 15026 \\
\hline
\end{tabular}

Tabla 2 Captura por comunidad de especies de la familia Formicidae de julio de 2008 a junio de 2009 en los tres hábitats de las comunidades Altuspata, Alto Choro, y Choro municipio de Coripata segunda sección de la provincia Nor Yungas del Departamento de La Paz, Bolivia

\begin{tabular}{ccccc}
\hline & \multicolumn{4}{c}{ Número de Especies } \\
\cline { 2 - 5 } Comunidad & \multicolumn{4}{c}{ hábitats } \\
& Bosque & $\begin{array}{c}\text { Borde de } \\
\text { Bosque }\end{array}$ & $\begin{array}{c}\text { Área de } \\
\text { Cultivo }\end{array}$ & Total \\
Altuspata & 26 & 25 & 21 & 73 \\
Choro Alto & 12 & 10 & 19 & 41 \\
Choro & 10 & 18 & 15 & 43 \\
Total & 49 & 53 & 55 & 157 \\
\hline
\end{tabular}


Tabla 2 Subfamilias y géneros de hormigas colectadas de julio de 2008 a junio de 2009 en los tres hábitats de las comunidades Altuspata, Alto Choro, y Choro municipio de Coripata segunda sección de la provincia Nor Yungas del Departamento de La Paz, Bolivia

\begin{tabular}{|c|c|c|c|c|c|}
\hline SUBFAMILIAS & TRIBU & GENERO & ESPECIES & SUBESPECIE & AUTORES \\
\hline \multirow{4}{*}{ Dolichoderinae } & Dolichoderini & Forelius sp1 & & & Emery 1888 \\
\hline & & Iridiomyrmex sp1 & & & Mayr 1862 \\
\hline & & Technomyrmex spl & & & \\
\hline & & Technomyrmex sp 2 & & & \\
\hline \multirow{7}{*}{ Ecitoninae } & Ecitonini & Eciton sp1 & burchellii & & Westwood 1842 \\
\hline & & Neivamyrmex sp 1 & emersoni & & Wheeler W M 1921 \\
\hline & & Nomamyrmex sp1 & esenbeckii & & Westwood 1842 \\
\hline & & Labidus sp3 & coecus & & Latreille 1802 \\
\hline & & Labidos sp2 & mars & & Forel 1912 \\
\hline & & Labidus spl & praedator & & Smith F 1858 \\
\hline & & Labidus sp4 & spininoides & & Emery 1890 \\
\hline \multirow{6}{*}{ Formicinae } & Camponotini & Camponotus sp1 & & & Mayr 1861 \\
\hline & & Camponotus sp2 & & & \\
\hline & & Camponotus sp 3 & & & \\
\hline & & Camponotus sp4 & & & \\
\hline & & Camponotus sp 5 & & & \\
\hline & Plagiolepidini & Prenolepis spl & & & \\
\hline \multirow{17}{*}{ Myrmicinae } & Attini & Acromyrmex spl & & & Goncalves 1961, Bolton 1995 \\
\hline & & Acromyrmex sp 2 & rogosus & & Smith 1858 \\
\hline & & Acromyrmex sp 3 & subterraneus & Subterraneus & Forel 1893 \\
\hline & & Cyphomyrmex spl & & & Mayr 1862 \\
\hline & & Trachymyrmex & & & Forel 1893 \\
\hline & Cephalotini & Cephalotes spl & & & Latreille 1802 \\
\hline & Cremastogastrini & Cremastogaster sp 1 & & & Agassiz 1846 \\
\hline & Dacetini & Strumigenys sp1 & & & Smith 1860 \\
\hline & Formicoxenini & Nesomyrmex sp1 & & & Kempf 1959 \\
\hline & Myrmicini & Hylomyrma sp1 & & & Forel 1912 \\
\hline & Pheidolini & Pheidole sp1 & & & Wilson 2003 \\
\hline & & Pheidole sp2 & & & \\
\hline & & Pheidole sp3 & & & \\
\hline & & Pheidole sp 4 & socrates & & Forel 1912 \\
\hline & & Pheidole sp 5 & & & Wilson 2003 \\
\hline & Solenopsidini & Solenopsis spl & & & Westwood 1840 \\
\hline & & Solenopsis sp2 & & & \\
\hline \multirow{9}{*}{ Ponerinae } & Ectatommini & Gnamptogenys spI & & & Roger 1863 \\
\hline & Ponerini & Hypoponera sp1 & & & Santschi 1938 \\
\hline & Ponerini & Ponera & & & Latreille 1804 \\
\hline & Odontomachini & Odontomachus spl & chelifer & & Latreille 1802 \\
\hline & Odontomachini & Odontomachus sp 2 & biumbonatus & & Brown 1976 \\
\hline & Odontomachini & Odontomachus sp 3 & yucatecus & & \\
\hline & Thaumathomyrmecini & Pachycondyla spl & cauta & & Mann 1922 \\
\hline & Thaumathomyrmecini & Pachycondyla sp2 & impressa & & Roger 1861 \\
\hline & Thaumathomyrmecini & Pachycondyla sp3 & harpax & & Fabricius 1804 \\
\hline \multirow{3}{*}{ Pseudomyrmecinae } & Pseudomyrmecini & Pseudomyrmex spl & & & Ward 1990 \\
\hline & Pseudomyrmecini & Pseudomyrmex spl & & & \\
\hline & Pseudomyrmecini & Pseudomyrmex sp1 & & & \\
\hline
\end{tabular}


Tabla 3 Subfamilias y géneros de hormigas colectadas de julio de 2008 a junio de 2009 en el hábitat Bosque de las comunidades Altuspata, Alto Choro, y Choro municipio de Coripata segunda sección de la provincia Nor Yungas del Departamento de La Paz, Bolivia

\begin{tabular}{|c|c|c|c|c|c|}
\hline \multirow[b]{3}{*}{ Especie } & \multicolumn{5}{|c|}{ Bosque } \\
\hline & \multirow{2}{*}{$\begin{array}{c}\text { Altuspata } \\
\mathrm{Nr}\end{array}$} & \multirow{2}{*}{$\frac{\text { Choro Alto }}{\mathrm{Nr}}$} & \multirow{2}{*}{$\frac{\text { Choro }}{\mathrm{Nr}}$} & \multirow{2}{*}{ Total } & \multirow{2}{*}{ Media \pm SD } \\
\hline & & & & & \\
\hline Labidus spininoides & 1250 & 374 & 280 & 1904 & $634.67 \pm 534.96$ \\
\hline Labidus praedator & 515 & 191 & 247 & 953 & $317.67 \pm 173.17$ \\
\hline Nesomyrmex esenbeckii & -- & -- & -- & -- & -- \\
\hline Camponotus sp.1 & 7 & 0 & 1 & 8 & $2.67 \pm 3.79$ \\
\hline Acromyrmex spl & 27 & 298 & 0 & 325 & $108.33 \pm 164.81$ \\
\hline Crematogaster sp.1 & 1 & 0 & 0 & 1 & $0.33 \pm 0.58$ \\
\hline Acromyrmex rogusos & 3 & 0 & 0 & 3 & $1.00 \pm 1.73$ \\
\hline Odontomachus chelifer & 16 & 0 & 5 & 21 & $7.00 \pm 8.19$ \\
\hline Camponotus sp.2 & 16 & 0 & 0 & 16 & $5.33 \pm 9.24$ \\
\hline Nesomyrmex & 1 & 0 & 0 & 1 & $0.33 \pm 0.58$ \\
\hline Pheidole sp.1 & 17 & 0 & 0 & 17 & $5.67 \pm 9.81$ \\
\hline Technomyrmex sp.1 & 1 & 0 & 0 & 1 & $0.33 \pm 0.58$ \\
\hline Technomyrmex sp.2 & 1 & 0 & 0 & 1 & $0.33 \pm 0.58$ \\
\hline Camponotus sp.3 & 15 & 1 & 1 & 17 & $5.67 \pm 8.08$ \\
\hline Forelius & 7 & 0 & 0 & 7 & $2.33 \pm 4.04$ \\
\hline Camponotus sp.4 & 31 & 0 & 0 & 31 & $10.33 \pm 17.90$ \\
\hline Camponotus sp.5 & 1 & 0 & 0 & 1 & $0.33 \pm 0.58$ \\
\hline Solenopsis sp.1 & 4 & 0 & 0 & 4 & $1.33 \pm 2.31$ \\
\hline Pheidole 3 & 2 & 0 & 0 & 2 & $0.67 \pm 1.15$ \\
\hline Prenolepis & 1 & 2 & 0 & 3 & $1.00 \pm 1.00$ \\
\hline Eciton burchellii & 3 & 0 & 84 & 87 & $29.00 \pm 47.66$ \\
\hline Labidos mars & 3 & 4 & 0 & 7 & $2.33 \pm 2.08$ \\
\hline Solenopsis sp.2 & 5 & 0 & 0 & 5 & $1.67 \pm 2.89$ \\
\hline Pheidole Socrates & 1 & 0 & 0 & 1 & $0.33 \pm 0.58$ \\
\hline Cephalotes & 1 & 0 & 1 & 2 & $0.67 \pm 0.58$ \\
\hline P. harpax & 1 & 0 & 0 & 1 & $0.33 \pm 0.58$ \\
\hline Neivamyrmex emersoni & -- & -- & -- & -- & -- \\
\hline Odontomachus yucatecus & -- & -- & -- & -- & -- \\
\hline P. impresa & 0 & 0 & 60 & 60 & $20.00 \pm 34.64$ \\
\hline Pseudomyrmex sp.1 & 3 & 0 & 0 & 3 & $1.00 \pm 1.73$ \\
\hline Hylomyrma & 0 & 2 & 0 & 2 & $0.67 \pm 1.15$ \\
\hline Trachymyrmex & 0 & 2 & 0 & 2 & $0.67 \pm 1.15$ \\
\hline \multicolumn{6}{|l|}{ Odontomachus biumbunatus } \\
\hline Pseudomyrmex sp.2 & 0 & 2 & 0 & 2 & $0.67 \pm 1.15$ \\
\hline Pseudomyrmex sp.3 & 0 & 2 & 0 & 2 & $0.67 \pm 1.15$ \\
\hline Cурhотугтех & 0 & 1 & 0 & 1 & $0.33 \pm 0.58$ \\
\hline Ponera & 0 & 2 & 0 & 2 & $0.67 \pm 1.15$ \\
\hline Acromymex subterraneus subterraneus & 0 & 0 & 2 & 2 & $0.67 \pm 1.15$ \\
\hline Gnaptogenys & -- & -- & -- & -- & -- \\
\hline Stromigynes & -- & -- & -- & -- & -- \\
\hline P. cauta & -- & -- & -- & -- & -- \\
\hline Hypoponera & -- & -- & -- & -- & -- \\
\hline Iridiomyrmex & 0 & 0 & 1 & 1 & $0.33 \pm 0.58$ \\
\hline Labidus coecus & -- & -- & -- & -- & -- \\
\hline Pheidole 5 & -- & -- & -- & -- & -- \\
\hline Total & 1933 & 881 & 682 & 3496 & $1165.33 \pm 672.22$ \\
\hline
\end{tabular}


Tabla 4 Subfamilias y géneros de hormigas colectadas de julio de 2008 a junio de 2009 en el hábitat Borde de Bosque de las comunidades Altuspata, Alto Choro, y Choro municipio de Coripata segunda sección de la provincia Nor Yungas del Departamento de La Paz, Bolivia

\begin{tabular}{|c|c|c|c|c|c|}
\hline \multirow[b]{3}{*}{ Especie } & \multicolumn{5}{|c|}{ Borde de Bosque } \\
\hline & \multirow{2}{*}{$\frac{\text { Altuspata }}{\mathrm{Nr}}$} & \multirow{2}{*}{$\begin{array}{c}\text { Choro Alto } \\
\mathrm{Nr}\end{array}$} & \multirow{2}{*}{$\frac{\text { Choro }}{\mathrm{Nr}}$} & \multirow{2}{*}{ Total } & \multirow{2}{*}{ Media \pm SD } \\
\hline & & & & & \\
\hline Labidus spininoides & 327 & 2075 & 1904 & 4306 & $1435.33 \pm 963.65$ \\
\hline Labidus praedator & 385 & 20 & 1777 & 2182 & $727.33 \pm 927.18$ \\
\hline Nesomyrmex esenbeckii & -- & -- & -- & -- & -- \\
\hline Camponotus sp.1 & 2 & 0 & 2 & 4 & $1.33 \pm 1.15$ \\
\hline Acromyrmex sp1 & 42 & 15 & 4 & 61 & $20.33 \pm 19.55$ \\
\hline Crematogaster sp.1 & 18 & 0 & 1 & 19 & $6.33 \pm 10.12$ \\
\hline Acromyrmex rogusos & 1 & 0 & 1 & 2 & $0.67 \pm 0.58$ \\
\hline Odontomachus chelifer & 4 & 1 & 26 & 31 & $10.33 \pm 13.65$ \\
\hline Camponotus sp.2 & 8 & 0 & 7 & 15 & $5.00 \pm 4.36$ \\
\hline \multicolumn{6}{|l|}{ Nesomyrmex } \\
\hline Pheidole sp.1 & 29 & 0 & 3 & 32 & $10.67 \pm 15.95$ \\
\hline Technomyrmex sp.1 & -- & -- & -- & -- & -- \\
\hline Technomyrmex sp.2 & -- & -- & -- & -- & -- \\
\hline Camponotus sp.3 & 4 & 0 & 10 & 14 & $4.67 \pm 5.03$ \\
\hline \multicolumn{6}{|l|}{ Forelius } \\
\hline Camponotus sp.4 & 5 & 0 & 15 & 20 & $6.67 \pm 7.64$ \\
\hline Camponotus sp. 5 & 1 & 0 & 0 & 1 & $0.33 \pm 0.58$ \\
\hline Solenopsis sp.1 & 4 & 0 & 2 & 6 & $2.00 \pm 2.00$ \\
\hline Pheidole 3 & 8 & 1 & 0 & 9 & $3.00 \pm 4.36$ \\
\hline Prenolepis & 0 & 0 & 2 & 2 & $0.67 \pm 1.15$ \\
\hline Eciton burchellii & 38 & 0 & 772 & 810 & $270.00 \pm 435.16$ \\
\hline Labidos mars & 14 & 0 & 0 & 14 & $4.67 \pm 8.08$ \\
\hline Solenopsis sp.2 & -- & -- & -- & -- & -- \\
\hline Pheidole Socrates & -- & -- & -- & -- & -- \\
\hline Cephalotes & -- & -- & -- & -- & -- \\
\hline P. harpax & 5 & 0 & 0 & 5 & $1.67 \pm 2.89$ \\
\hline Neivamyrmex emersoni & -- & -- & -- & -- & -- \\
\hline Odontomachus yucatecus & -- & -- & -- & -- & -- \\
\hline P. impresa & 0 & 12 & 0 & 12 & $4.00 \pm 6.93$ \\
\hline Pseudomyrmex sp.1 & 2 & 0 & 2 & 4 & $1.33 \pm 1.15$ \\
\hline Hylomyrma & 4 & 0 & 2 & 6 & $2.00 \pm 2.00$ \\
\hline Trachymyrmex & 1 & 0 & 0 & 1 & $0.33 \pm 0.58$ \\
\hline Odontomachus biumbunatus & 2 & 1 & 0 & 3 & $1.00 \pm 1.00$ \\
\hline Pseudomyrmex sp.2 & 1 & 0 & 10 & 11 & $3.67 \pm 5.51$ \\
\hline Pseudomyrmex sp.3 & 2 & 0 & 0 & 2 & $0.67 \pm$ \\
\hline Cyphomyrmex & 2 & 0 & 0 & 2 & $0.67 \pm 1.15$ \\
\hline Ponera & -- & -- & -- & -- & -- \\
\hline Acromymex subterraneus subterraneus & -- & -- & -- & -- & -- \\
\hline Gnaptogenys & 0 & 1 & 0 & 1 & $0.33 \pm 0.58$ \\
\hline Stromigynes & 0 & 2 & 0 & 2 & $0.67 \pm 1.15$ \\
\hline$P$. cauta & 0 & 1 & 0 & 1 & $0.33 \pm 0.58$ \\
\hline Hypoponera & 0 & 0 & 1 & 1 & $0.33 \pm 0.58$ \\
\hline Iridiomyrmex & -- & -- & -- & -- & -- \\
\hline Labidus coecus & -- & -- & -- & -- & -- \\
\hline Pheidole 5 & -- & -- & -- & -- & -- \\
\hline Total & 909 & 2129 & 4541 & 7579 & $2526.33 \pm 1848.31$ \\
\hline
\end{tabular}


Tabla 4 Subfamilias y géneros de hormigas colectadas de julio de 2008 a junio de 2009 en el hábitat Área de cultivo de las comunidades Altuspata, Alto Choro, y Choro municipio de Coripata segunda sección de la provincia Nor Yungas del Departamento de La Paz, Bolivia

\begin{tabular}{|c|c|c|c|c|c|}
\hline \multirow[b]{3}{*}{ Especie } & \multicolumn{5}{|c|}{ Área de Cultivo } \\
\hline & \multirow{2}{*}{$\frac{\text { Altuspata }}{\mathrm{Nr}}$} & \multirow{2}{*}{$\frac{\text { Choro Alto }}{\mathrm{Nr}}$} & \multirow{2}{*}{$\begin{array}{c}\text { Choro } \\
\mathrm{Nr} \\
\end{array}$} & \multirow{2}{*}{ Total } & \multirow{2}{*}{ Media \pm SD } \\
\hline & & & & & \\
\hline Labidus spininoides & 9 & 366 & 16 & 391 & $130.33 \pm 204.12$ \\
\hline Labidus praedator & 1628 & 479 & 775 & 2882 & $960.67 \pm 59658$ \\
\hline Nesomyrmex esenbeckii & 2 & 0 & 0 & 2 & $0.67 \pm 1.15$ \\
\hline Camponotus sp.1 & 19 & 2 & 7 & 28 & $9.33 \pm 8.74$ \\
\hline Acromyrmex sp1 & 2 & 0 & 0 & 2 & $0.67 \pm 1.15$ \\
\hline Crematogaster sp.1 & 6 & 0 & 0 & 6 & $2.00 \pm 3.46$ \\
\hline Acromyrmex rogusos & 0 & 3 & 0 & 3 & $1.00 \pm 1.73$ \\
\hline Odontomachus chelifer & 21 & 41 & 5 & 67 & $22.33 \pm 18.04$ \\
\hline Camponotus sp.2 & 41 & 15 & 2 & 58 & $19.33 \pm 19.86$ \\
\hline Nesomyrmex & -- & -- & -- & -- & -- \\
\hline Pheidole sp.1 & 9 & 92 & 32 & 133 & $44.33 \pm 42.85$ \\
\hline Technomyrmex sp.1 & -- & -- & -- & -- & -- \\
\hline Technomyrmex sp.2 & -- & -- & -- & -- & -- \\
\hline Camponotus sp.3 & 9 & 2 & 0 & 11 & $3.67 \pm 4.73$ \\
\hline Forelius & 6 & 0 & 0 & 6 & $2.00 \pm 3.46$ \\
\hline Camponotus sp.4 & 15 & 0 & 0 & 15 & $5.00 \pm 8.66$ \\
\hline Camponotus sp.5 & 4 & 0 & 0 & 4 & $1.33 \pm 2.31$ \\
\hline Solenopsis sp.1 & -- & -- & -- & -- & -- \\
\hline Pheidole 3 & 15 & 21 & 1 & 37 & $12.33 \pm 10.26$ \\
\hline Prenolepis & -- & -- & -- & -- & -- \\
\hline Eciton burchellii & 0 & 1 & 32 & 33 & $11.00 \pm 18.19$ \\
\hline Labidos mars & 10 & 0 & 0 & 10 & $3.33 \pm 5.77$ \\
\hline Solenopsis sp.2 & -- & -- & -- & -- & -- \\
\hline Pheidole Socrates & 4 & 0 & 0 & 4 & $1.33 \pm 2.31$ \\
\hline Cephalotes & -- & -- & -- & -- & -- \\
\hline P. harpax & 10 & 55 & 0 & 65 & $21.67 \pm 29.30$ \\
\hline Neivamyrmex emersoni & 4 & 5 & 1 & 10 & $3.33 \pm 2.08$ \\
\hline Odontomachus yucatecus & 10 & 21 & 18 & 49 & $16.33 \pm 5.69$ \\
\hline P. impresa & 4 & 72 & 1 & 77 & $25.67 \pm 40.15$ \\
\hline Pseudomyrmex sp.1 & -- & -- & -- & -- & -- \\
\hline Hylomyrma & -- & -- & -- & -- & -- \\
\hline Trachymyrmex & -- & -- & -- & -- & -- \\
\hline Odontomachus biumbunatus & 0 & 40 & 12 & 52 & $17.33 \pm 20.53$ \\
\hline Pseudomyrmex sp.2 & -- & -- & -- & -- & -- \\
\hline Pseudomyrmex sp.3 & -- & -- & -- & -- & -- \\
\hline Cyphomyrmex & -- & -- & -- & -- & -- \\
\hline Ponera & -- & -- & -- & -- & -- \\
\hline Acromymex subterraneus subterraneus & -- & -- & -- & -- & -- \\
\hline Gnaptogenys & 0 & 1 & 0 & 1 & $0.33 \pm 0.58$ \\
\hline Stromigynes & 0 & 2 & 0 & 2 & $0.67 \pm 1.15$ \\
\hline P. cauta & 0 & 1 & 0 & 1 & $0.33 \pm 0.58$ \\
\hline Hypoponera & -- & -- & -- & -- & -- \\
\hline Iridiomyrmex & -- & -- & -- & -- & -- \\
\hline Labidus coecus & 0 & 0 & 1 & 1 & $0.33 \pm 0.58$ \\
\hline Pheidole 5 & 0 & 0 & 1 & 1 & $0.33 \pm 0.58$ \\
\hline Total & 1828 & 1219 & 904 & 3951 & $1317.00 \pm 469.73$ \\
\hline
\end{tabular}


Fig 1 Abundancia relativa de hormigas colectadas de julio de 2008 a junio de 2009 en los tres hábitats de las comunidades Altuspata, Alto Choro, y Choro municipio de Coripata segunda sección de la provincia Nor Yungas del Departamento de La Paz, Bolivia

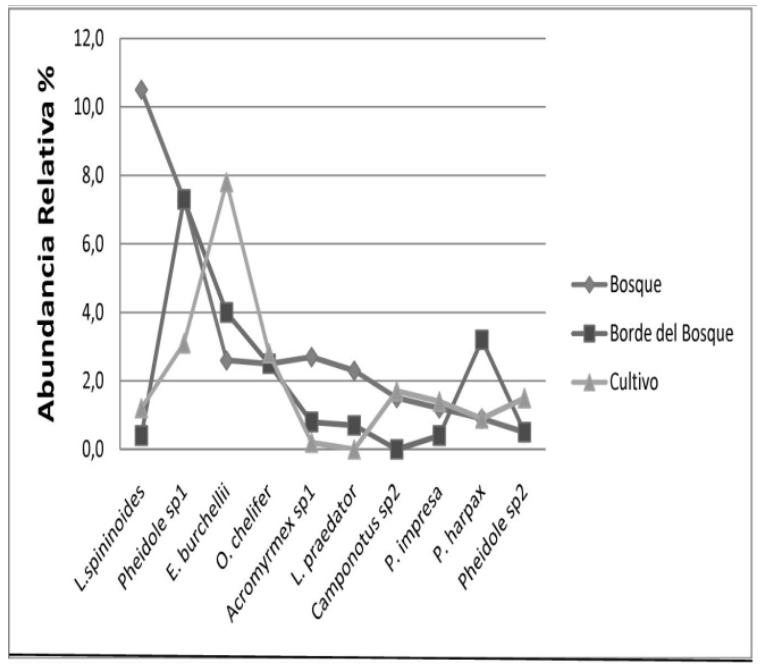

Fig 2 Análisis Clúster basado en los 3 hábitats de julio de 2008 a junio de 2009 capturadas en la trampa de caída (pitfall), en las comunidades Altuspata, Alto Choro, y Choro municipio de Coripata segunda sección de la provincia Nor Yungas del Departamento de La Paz, Bolivia

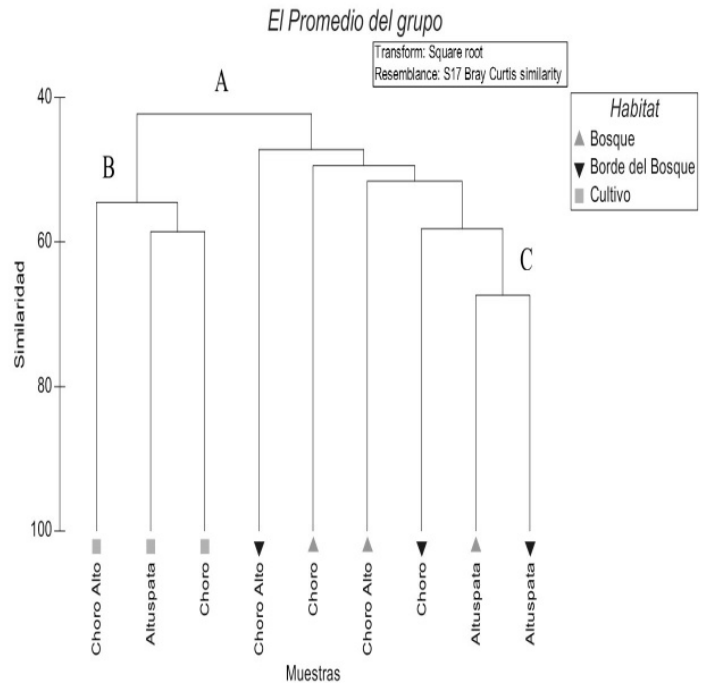

Fig 3 Análisis de escala multi-dimensional para el factor hábitat de julio de 2008 a junio de 2009 de las comunidades Altuspata, Alto Choro, y Choro capturadas en la trampa de caída (pitfall), municipio de Coripata segunda sección de la provincia Nor Yungas del Departamento de La Paz, Bolivia

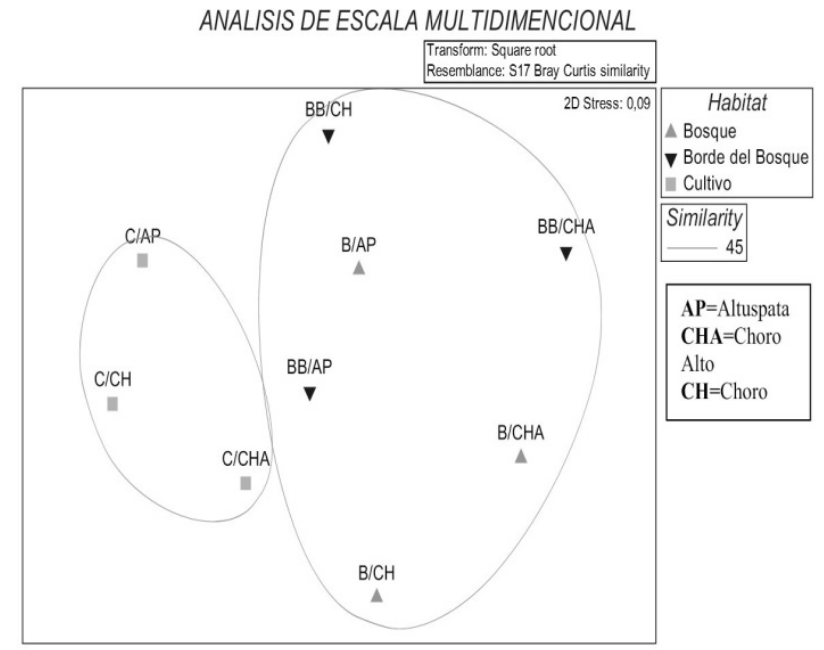

Tabla 3 Análisis de similaridad de abundancia para el factor hábitat por de adultos de la familia Formicidae de julio de 2008 a junio de 2009 de las comunidades Altuspata, Alto Choro, y Choro municipio de Coripata segunda sección de la provincia Nor Yungas del Departamento de La Paz, Bolivia

\begin{tabular}{|c|c|c|c|c|c|}
\hline \multicolumn{6}{|l|}{ Prueba Global } \\
\hline \multicolumn{6}{|c|}{ Muestra Estadística (Global R): 0,169 } \\
\hline \multicolumn{6}{|c|}{ Nivel de significancia de la muestra estadística: $78,9 \%$} \\
\hline \multicolumn{6}{|c|}{ Cantidad de permutaciones: 280 (Permutaciones posibles ) } \\
\hline \multicolumn{6}{|c|}{ Número de las estadísticas igual o superior a Global R: 59} \\
\hline \multicolumn{6}{|l|}{ Prueba de pares } \\
\hline Grupos & Valor de $\mathbf{R}$ & $\begin{array}{l}\% \text { nivel de } \\
\text { significancia }\end{array}$ & $\begin{array}{l}\text { Permut } \\
\text { aciones } \\
\text { posibles }\end{array}$ & $\begin{array}{l}\text { Permutaci } \\
\text { ón actual }\end{array}$ & $\begin{array}{c}\text { Números }>= \\
\text { observados }\end{array}$ \\
\hline \multirow{2}{*}{$\begin{array}{l}\text { Bosque, Borde } \\
\text { del Bosque } \\
\text { Bosque, Cultivo }\end{array}$} & -0.370 & 0 & 10 & 10 & 10 \\
\hline & 0.481 & 90 & 10 & 10 & 1 \\
\hline $\begin{array}{l}\text { Borde del } \\
\text { Bosque, Cultivo }\end{array}$ & 0.481 & 90 & 10 & 10 & 1 \\
\hline
\end{tabular}


Fig 4 Análisis de contribución de morfoespecies de la familia Formicidae a la semejanza para el factor hábitat de julio de 2008 a junio de 2009 de las comunidades Altuspata, Alto Choro, y Choro capturadas en la trampa de caída (pitfall), municipio de Coripata segunda sección de la provincia Nor Yungas del Departamento de La Paz, Bolivia

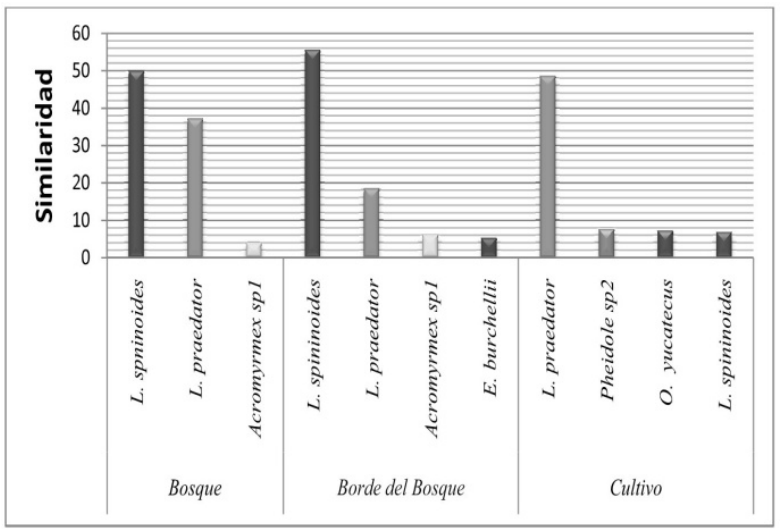

Fig 5 Análisis de contribución de morfoespecies de la familia Formicidae a la disimilitud para el factor hábitat de julio de 2008 a junio de 2009 de las comunidades Altuspata, Alto Choro, y Choro capturadas en la trampa de caída (pitfall), municipio de Coripata segunda sección de la provincia Nor Yungas del Departamento de La Paz, Bolivia

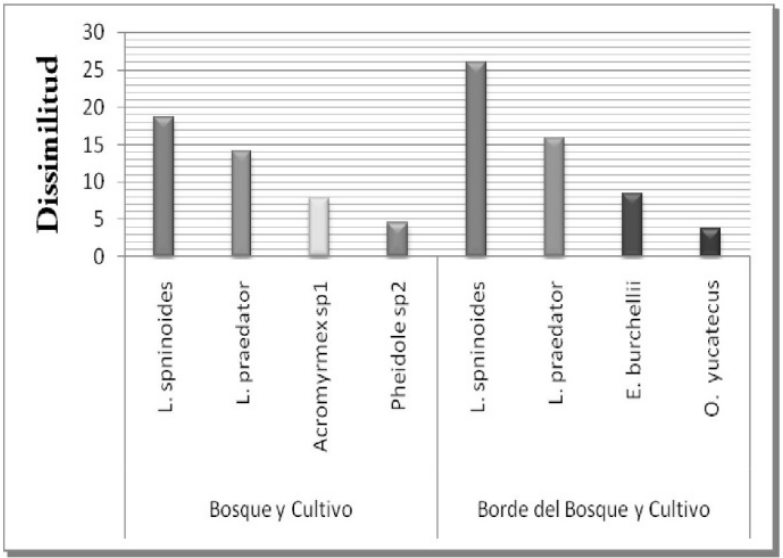

Fig 6 Curva de acumulación de morfoespecies de la familia Formicidae de julio de 2008 a junio de 2009 de las comunidades Altuspata, Alto Choro, y Choro capturadas en la trampa de caída (pitfall), municipio de Coripata segunda sección de la provincia Nor Yungas del Departamento de La Paz, Bolivia

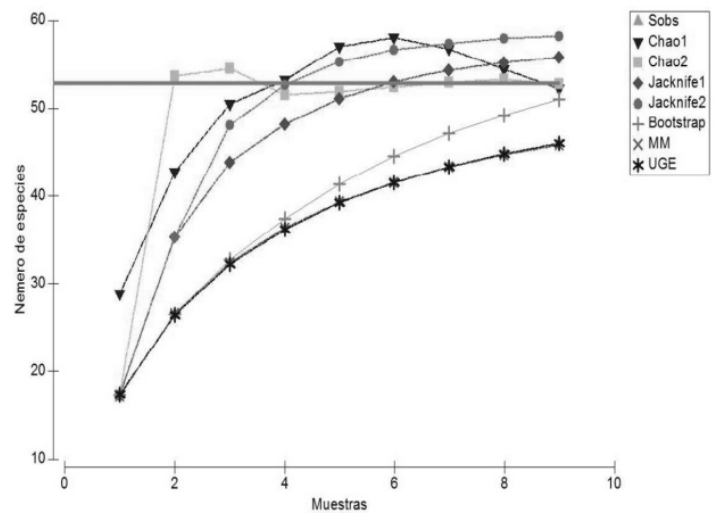

\section{Discusión}

La subfamilia Ecitoninae representa una riqueza y abundancia con dos especies Labidus spininodis (Emery 1890) y Labidus praedator (Smith F 1858), la subfamilia Dolichoderinae con el género Forelius (Emery 1888) fueron capturadas en bosque, borde de bosque y área de cultivo en las comunidades del Choro, Alto Choro y Altuspata en mayor número de individuos con relación a las otras especies, siendo que estas presentan hábitos depredadores (Palacio 2003).

La subfamilia Myrmicinae representa con mayor número en individuos a la especie Acromyrmex sp1 siendo de hábitos fitófago, esto debido a la vegetación existente en los sitios (Della Lucía 2003, Delabie et al 2003). Esta especie recoge las plantas para cultivar hongos que ellos consumen, o sea entre esta especie de hormiga y el hongo existe una relación simbiótica. Otro género que presenta 
número mayor es Pheidole (Wilson 2003) de la misma subfamilia.

Las especies Odontomachus chelifer (Latreille 1802) y Pachyconcyla impressa (Roger 1861) son cazadores solitarios de la subfamilia Ponerinae. Ellos demuestran un aporte en la cantidad de individuos capturados en los hábitats de estudio. Las especies del género Pseudomyrmex (Ward 1990) (Pseudomyrmecinae) presentaron una menor cantidad de individuos, esta especie se capturó en bosque y borde del bosque, estas subfamilias viven en estratos arbóreos nidificando dentro de las ramas muertas, de los bosques tropicales (Ward 2003).

La subfamilia Myrmicinae es considerada dentro de la mirmecofauna como la más rica a nivel mundial, nidifican en todos los estratos y micro hábitats disponibles en los bosques lluviosos donde han sufrido una mayor diversificación a pesar de las diferencias altitudinales (Fernández et al 1996).

En la presente investigación se obtuvo mayor cantidad de géneros y especies en la subfamilia Myrmicinae (Rivera \& Ambrecht 2005) registraron y calcularon la riqueza de Myrmicinae, que encontró distribuida ampliamente a nivel de especie. Fernández 2003a, Myrmicinas ocupan cerca de la mitad de la fauna existente en términos de biomasa en algunos ecosistemas, (Vergara Navarro et al 2007), la subfamilia Myrmicinae es superior con respecto a otras subfamilias agrupando la mayor cantidad de especies con diferentes hábitos de alimentación.

Díaz et al (2009) en su trabajo encontró mayor cantidad de géneros que alcanzó a 50\% del número total de especies encontradas de la subfamilia Myrmicinae, son habitantes del suelo y hojarasca, también existen especies de formas arborícolas (Rivera \& Ambrecht 2005).

La tabla 1 muestra un total de 15026 individuos de la familia Formicidae capturadas en los hábitats estudiados, Choro presenta 10 especies, Choro Alto 12, Altuspata 26 especies, especies en bosque, en tanto en borde de bosque se registraron 18,10 y 25 respectivamente, quizá la variación altitudinal (Amat et al 1999, Fagua 1999) en Bogotá, Colombia, reportan un efecto en la riqueza de mariposas y hormigas por la altitud, indican que a medida que incrementa la altura se reduce la riqueza, aumentando la dominancia de algunas especies, (Castro Delgado et al 2008) la disminución de la riqueza en forma lineal. Lattke (2003a) expresa que hay disminución de riqueza debido al aumento de altitud, relacionándolo con bajas temperaturas de las tierras altas así mismo baja las actividades físicas y procesos fisiológicos. Sin embargo, el suministro de agroquímicos en los alrededores de un bosque reduce la diversidad de especies por el proceso de volatilización (Marcot et al 2000), la existencia de cultivos de coca cerca del área de bosque y borde del bosque tenga una influencia en la población captura.

En bosque, la especie Labidus spininoides aporta mayor porcentaje de abundancia debido a las características predadores que tienen esta especie, viven en grupo, las colonias caminan en grupo y tiene una colonia compuesta de numerosas integrantes (Palacio 2003).

En el borde del bosque Pheidole sp1, presenta 
mayor abundancia, Fernández (2003a) indica que es habitante principalmente de estrato epigeo, localmente presenta mayor abundancia, posiblemente por la misma composición de vegetación y la distribución en el ecosistema. Vergarra Navarro et al (2007) indican que entre las plantas y hormigas existe una relación facultativa haciendo alguna de sus colonias en árboles, raíces, es diferente el sustrato que hay en la corona hay la relación con alguno insectos de orden Hemíptera, homóptera y otros. Estarían de visita en esta área por sus hábitos de alimentación, pues las hormigas se trasladan largas distancias en busca de su alimento, ya sea insectos como presas o vegetales, cuanto mayor la diversidad de vegetales mayor riqueza de hormigas.

En el área de cultivo, Eciton burchellii (Westwood 1842) presenta mayor abundancia, (Palacio 2003) las hormigas de esta subfamilia Ecitoninae son predominantes en su distribución, gran número de obreras que integra su colonia, genero donde se encontró mayor número de individuos, llegan a alcanzar hasta tres millones.

En el análisis de clúster, existe una semejanza de $43 \%$ entre las comunidades y hábitats (A), además, Altuspata tiene una similaridad de $65 \%$ entre bosque y borde de bosque $(\mathrm{C})$ que presenta el mismo microclima y estructura vegetal, Choro y Altuspata con $58 \%$ de similaridad con área de cultivo, sumándose con Choro Alto con 53\% (B), esta área de cultivo tiene las mismas condiciones de los tres hábitats de estudio.

El análisis de escala multi-dimensional, Fig 3, para comparar las semejanzas basadas en el índice de Bray-Curtis.

37
La agrupación del área del cultivo es muy claramente separada de los demás hábitats (A), lo que se debe a la homogeneidad del cultivo de coca (Erythroxylum coca), el borde del bosque y bosque de Choro Alto están separados de los demás sitios (B) probablemente la altitud, otros habitas en los lugares más altos. Andersen (1995, 1997) señala que la composición y dominancia de grupos funcionales varía entre zonas climáticas y dentro de una zona en particular varía sistemáticamente con el tipo de vegetación.

El análisis de similaridad en la prueba de pares muestra que existe una probabilidad de $78.9 \%$ de diferencias entre hábitats en cuanto a la riqueza en la comunidad de mirmecofauna, es decir, existe una probabilidad mayor a $78.9 \%$ de que las especies encontradas en los hábitats sean diferentes. Entre el bosque y el cultivo, el borde del bosque y cultivo, presenta una probabilidad de 90\% de diferencias, indicando que la composición de especies es diferente de áreas cultivo frente a los bosques y los bordes de bosque.

Entre borde del bosque y bosque no existen diferencias (nivel de significancia de $0 \%$ ), indicando que las hormigas se asemejen entre estos hábitats.

Lozano Zambrano et al (2009) comentan que hay relaciones entre la riqueza de especies con el hábitat y el aumento de población de hormigas en los bosques pequeños ocasiona el efecto borde siendo especies generalistas, (Peña-Becerril et al 2005) la riqueza de especies aumenta con el área de vegetación natural en los bosques tropicales. 
En el bosque se observa de que Labidus spininoides contribuyó a la semejanza entre sitios con un porcentaje de $49.83 \%$, seguida del Labidus praedator con un $37.06 \%$ de contribución El área de bosque presentó condiciones adecuadas para esta especie, el área borde del bosque con Labidus spininoides muestra un mayor aporte de (55.47\%) seguido Labidus praedator siendo esta área la que contribuye mayor con la especie Labidus spininoides en comparación con los otros hábitats por la zona de transición que presenta y donde están una variedad de presas que son víctimas de esta especie Acromyrmex sp1 presenta 5.96\% y Eciton burchellii con 5.15\%de contribución de semejanza entre sitios. Esta última especie tiene preferencia en su hábito de lugares con hojarascas que pertenece al grupo de hormigas legionarias (Lozano et al 2009).

En el área de cultivo Labidus praedator aporta a la similaridad entre sitios con $48.49 \%$ por la característica misma de especie que pertenece a las depredadoras legionarias. Esta subfamilia depreda todo a su paso y no tiene preferencias alimentarias. Probablemente sus presas sean plagas fitófagas ya que en el área de cultivo están más las plagas y algunos insectos benéficos.

En las dos áreas es notorio el aporte de la contribución a la similaridad de las especies Labidus spininodis y Labidus praedator, estas especies tienen preferencias en obtención de alimentos, actúan de forma legionaria; esta es una de las características más notorias de la subfamilia Ecitoninae. El género Labidus de la dicha subfamilia no construye sus colonias permanentes ni está mucho tiempo en un solo lugar. Son hormigas depredadoras de hábitos nómadas, tiene ciclo de vida alterna con fases estacionarias, migratorias y arrasa todo lo que encuentra a su paso y son famosas en áreas rurales limpiando casas (Palacio 2003).

El género Acromyrmex sp1se presenta también en las dos áreas en cantidades menores en comparación en el género Labidus. Esta especie es considerada podadora o cortadora: lleva las partes de las plantas a sus hormigueros un vez cortada donde preparan un sustrato para cría de hongos que sirve para su alimentación esto causa grandes daños en la agricultura (Vaccaro \& Mousques 1997).

Al par del análisis de similaridad, se realiza también un análisis de disimilaría de morfoespecies (Fig 4). A continuación se muestra la comparación del aporte a la disimilaridad de morfoespecies comparando el área de cultivo con el bosque y el borde del bosque. Se realizaron estas dos comparaciones solamente por la significancia obtenida (tabla 3 ).

La especie Labidus spininoides contribuyó a la disimilitud con mayor aporte en los grupos conformados. Los resultados en la comparación entre bosque y cultivo es el Labidus spininoides con $18.58 \%$, con $14.04 \%$, el Labidus praedator con $7.8 \%$, el Acromyrmex sp1 y el Pheidole sp2con $4.52 \%$ lo que explica que en bosque hay gran diversidad de especies presentes.

El bosque presentó a las especies Labidus praedator debido a la nidificación de comportamiento legionaria y dieta ocasional (Kattan et al 2008, Palacio 1999), y Pheidole sp2 por relaciones que tiene con vegetación Lozano Zambrano et al (2009): mencionan que son 
habitantes de hojarasca las dos especies de Labidus praedator y Pheidole sp2. Sin esta especie rara vez se alimenta de materia vegetal y sólo si sus presas están presentes en esa área (Palacio 1999), y el último aporte de la especie Acromyrmex sp1 de preferencias fitófagos.

Los hábitats de borde del bosque y cultivo contribuyen con Labidus spininoides con 25,94\% seguido con la Labidus praedator con 15,81\%, Eciton burchellii con $8,41 \%$ y la Odontomachus yucatecus con $3,74 \%$. Son las especies que contribuyeron a la disimilitud entre estos hábitats puesto que el borde del bosque fue la que tuvo mayor variedad de especies y en el cultivo. Debido a que esta especie se moviliza en columnas formada de varias obreras integrantes de la colonia (Palacio 2003).

La zona de los yungas es nominada ceja de monte yungueño y está dentro del corredor biológico bioceánico, tiene la característica de presentar alta diversidad de especies tanto en fauna y flora (Alarcón 2008).

En Tariquía (Tarija, Bolivia) en un bosque seco, Limachi (2006) registró 137 morfoespecies distribuidas en 29 géneros pertenecientes a 6 subfamilias que son las mismas subfamilias que se encontraron en el presente trabajo, aunque el lugar de estudio es diferente por la altura y clima. Vergara Navarro et al (2007), al determinar la riqueza y composición de hormigas en una zona de bosque húmedo pre montano tropical, registró 55 especies y 28 géneros distribuidos en 7 subfamilias. Estos resultados son similares a los resultados obtenidos en el presente trabajo.
La presente investigación muestra la gran riqueza y abundancia de hormigas que existen entre comunidades y hábitats. Es muy diversa el grupo de mirmecofauna, sin embargo en trabajos similares con las mismas características topográficas y altura sobre el nivel del mar se encontraron cantidades similares. Se obtuvieron 46 especies o morfoespecies de hormigas en el presente trabajo.

En sí que los bosques albergan los hábitats o refugios de la entomofauna haciendo una importancia de relación entre bosques ya que las hormigas cumplan funciones importantes por su relación con la vegetación, hongos e insectos homópteros, cumpliendo roles de importancia para un aprovechamiento y conservación de este recurso. A pesar que, las hormigas tienen diferentes hábitos como: parasitoides, depredadores, fitófagos o plagas, pocas tienen un hábito homogéneo.

Finalmente las hormigas presentan una variación entre hábitats (bosque, borde del bosque $\mathrm{y}$ cultivo), pero no entre épocas ni comunidades, en el área estudiada dentro de los bosques premontanos tropicales de los yungas.

\section{Conflictos de interés}

Esta investigación recibió financiamiento de Benson Agriculture and Food Institute y Brigham Young University (B.Y.U) y no presenta conflictos de interés. 


\section{Agradecimientos}

Los autores agradecen a los productores de las comunidades de Choro, Alto Choro, y Altuspata por permitir el desarrollo de este trabajo, al personal del Laboratorio de Entomología de la Carrera de Ingeniería Agronómica de la Unidad Académica Campesina Carmen Pampa. A Benson Agriculture and Food Institute y Brigham Young University (B.Y.U) por el financiamiento de esta investigación.

\section{Literatura citada}

Acosta Y, Cayama J, Gómez E, Reyes N, Rojas D, García H.Respiración microbiana y prueba de fitotoxicidad en el proceso de compostaje de una mezcla de residuos orgánicos. Multiciencias. 2006; 6:220-227.

Alarcón E. Diversidad de insectos de las familias Syrphidae y Carabidae en tres nichos ecológicos (bosque, borde del bosque y área del cultivo), de tres comunidades (San Juan de la Miel, San Pablo y Carmen Pampa) del municipio de Coroico. Nor Yungas de La Paz. Tesis Licenciatura. Unidad Académica Campesina Carmen Pampa. Universidad Católica Boliviana. La Paz, Bolivia, 2008. 96 pp.

Amat GG, Andrade CGM, Fernández, F. Insectos de Colombia. Santa Fé de Bogotá, Academia Colombiana de Ciencias Exactas, Físicas y Naturales. 1999; 434 pp.

Andersen AN. Functional groups and patterns of organization in North American ant communities: a comparison with Australia. Journal of Biogeography. 1997; 24:433-460.
Andersen AN. A classification of Australian ant communities based on functional group which parallel plant life-forms in relation to stress and disturbance. Journal of Biogeography. 1995; 20:15-29.

Baroni-Urbani C. Clave para la identificación de los géneros de hormigas neotropicales. Revista de Entomólogos Ibéricos (Basel). 1983; 9:7382.

Bolton B, Holldobler B, Wilson E. A key to the ant genera of Central and South America, the Indies, and lowland tropical Mexico. 1990; 140 pp.

Bolton B. Identification guide to the ant genera of the world. Cambridge, Harvard University Press. 1994; 152 pp.

Brown WL Jr. Contributions toward a reclassification of the Formicidae. V. Ponerinae, Tribes Platythyreini, Cerapachyini, Cylindromyrmicini, Acanthostichini, and Aenictogitini. New York, Cornell University Agricultural Experiment Station. 1963; 115 pp.

Castro Delgado S, Vergara C, Arrellano C. Distribución de la riqueza, composición taxonómica y grupos funcionales de hormigas del suelo a lo largo de un gradiente altitudinal en refugio de vida silvestre Laquipampa, Lambayeque-Perú. Lima, Departamento Académico de Biología, Universidad Nacional Agraria “La Molina”. 2008; (1)89-103.

Clarke KR, Warwick RM. Change in marine communities: an approach to statistical analysis and interpretation. 2 ed. Plymouth, RU, PRIMER-E Ltd. 2001; 176 pp.

Delabie JHC, Ospina M, Zabala G. Relaciones entre hormigas y plantas: una introducción. 2003; 167-180pp. En: Fernández, F. (ed.), Introducción a las hormigas de la región 
neotropical. Bogotá, Instituto de Investigación de Recursos Biológicos Alexander von Humboldt.

Della Lucía TMC. Hormigas de importancia económica en la región Neotropical. 2003; 338-349pp. En: Fernández, F. (ed.), Introducción a las hormigas de la región neotropical. Bogotá, Instituto de Investigación de Recursos Biológicos Alexander von Humboldt.

Díaz JA, Molano CE, Gaviria JC. Diversidad genérica de hormigas (Hymenoptera: Formicidae) en ambientes de bosque seco de los montes de María. Programa de biología. Universidad de Sucre, Colombia. Rev Colombiana Cien Anim. 2009; 1(2):279-285.

Fagua G. Variación de las mariposas y hormigas en un gradiente altitudinal de la Cordillera Oriental (Colombia). 1999; 317-362pp. En: Amat G., G; Andrade C., GM; Fernández, F, Insectos de Colombia. Bogotá, Academia Colombiana de Ciencias Exactas, Físicas y Naturales.

Fernandéz F, Sharkey MJ. Introducción a los Hymenoptera de la Región Neotropical. Sociedad Colombiana de Entomología, 2006. Bogotá D. C.

Fernandéz F, Sendoya S. List of Neotropical Ants (Himenóptera: Formicidae). Revista Biota Colombiana. 2004; 5(1);3-93.

Fernández $\mathrm{F}$ (ed.). Breve introducción a la biología social de las hormigas. 2003a; 81-96 pp. En: Fernández, F. (ed.), Introducción a las hormigas de la región neotropical. Bogotá, Instituto de Investigación de Recursos Biológicos Alexander von Humboldt.

Fernández $F$ (ed.). Sub familia Myrmicinae. 2003b, 307-330 pp. En: Fernández, F. (ed.), 41
Introducción a las hormigas de la región neotropical. Bogotá, Instituto de Investigación de Recursos Biológicos Alexander von Humboldt.

Fernández F, Palacio E, MacKay WP, MacKay V. Introducción al estudio de las hormigas (Hymenoptera: Formicidae) de Colombia. 1996, 351-416 pp. En: MG Andrade; GA Fernández (eds.), Insectos de Colombia, estudios escogidos. Bogotá, Academia Colombiana de Ciencias Exactas Físicas y Naturales. Colección Jorge Álvarez Lleras No10, Coedición con el Centro Editorial Javeriano.

Gauld I, Bolton B. (Eds.). The Hymenoptera. Oxford University Press. 1988. Great Britain.

Gobierno Municipal de Coripata. Plan de Desarrollo Municipal (PDM). 2006-2010. Segunda Sección de la Provincia Nor Yungas. La Paz, Bolivia: Servicios, Proyectos e inversiones COSEPI; 2006.

Holldobler B, Wilson EO. The Ants. Belknap press of Harvard University Press, Cambridge, Massachusetts, USA. 1990.

Kattan GH, Murcia C, Aldana RC Usma S. Relaciones entre hormigas y melastomatáceas en un bosque lluvioso del pacífico colombiana. Museo de Entomología de la Universidad del Valle. Colombia. 2008; 1:10.

LaSalle J, Gauld ID. Hymenoptera: their diversity, and their impact on the diversity of other organisms. 1993; 1-26 pp. IN: LaSalle J. and I.D. Gauld (Eds.). Hymenoptera and biodiversity. CABI. United Kingdom.

LaSalle J. Parasitic Hymenoptera, biological control and biodiversity. 1993; 197-215 pp. En: LaSalle J. and I.D. Gauld (Eds.), Hymenoptera and biodiversity. CABI. United Kingdom. 
Lattke JE. Clave para la determinación de hormigas neotropicales basados en las obreras. 2004; 117-148 pp. En: K. Jaffé (ed.). El mundo de las hormigas. Caracas, Ediciones de la Universidad Simón Bolívar.

Lattke JE. Conservación de una colección de hormigas. 2003a; 211-218 pp. En: Fernández, F (ed.), Introducción a las hormigas de la región neotropical. Bogotá, Instituto de Investigación de Recursos Biológicos Alexander von Humboldt.

Lattke JE. Distribución de las hormigas neotropicales. 2003b; 65-85 pp. En: Fernández F (ed.), Introducción a las hormigas de la región neotropical. Bogotá, Instituto de Investigación de Recursos Biológicos Alexander von Humboldt.

Limachi KM. Estructura y diversidad de hormigas en tres tipos de bosque alto de la reserva nacional de flora y fauna Tariquía. Tarija. Colección Boliviana de Fauna. 2006; 102 pp.

Lozano Zambrano F, Ulloa Chacón P, Armbrecht I. Ecology, behavior and bionomics: hormigas: relaciones especies-área en fragmentos de bosque seco tropical. Bogotá, Instituto Humboldt. 2009; 54 pp.

Marcot BG, Rumiz DI, Fredericksen TS. 2000. Definición de redes de áreas protegidas forestales: un manual para la delimitación de áreas protegidas forestales en los bosques manejados de las tierras bajas de Bolivia. Santa Cruz, Chemonics International, USAID/Bolivia. 2000; 19 pp.

Palacio E, Fernández F. Clave para las subfamilias y géneros. 2003; 221-249 pp. En: Fernández, F (ed.), Introducción a las hormigas de la región neotropical. Bogotá, Instituto de Investigación de Recursos Biológicos Alexander von
Humboldt.

Palacio EE. Subfamilia Ecitoninae. 2003; 281-283 pp. En: Fernández, F. (ed.), Introducción a las hormigas de la región neotropical. Bogotá, Instituto de Investigación de Recursos Biológicos Alexander von Humboldt.

Palacio E. Hormigas legionarias (Hymenoptera: Formicidae: Ecitoninae) de Colombia. 1999; 117-152 pp. En: Amat G, G; Andrade C, GM; Fernandez, F. 1999. Insectos de Colombia. Bogotá, Academia Colombiana de Ciencias Exactas, Físicas y Naturales. 434 pp.

Peña-Bicerril JC, Monroy-Ata A, Álvares-Sánchez FJ, Orozco-Almanza SM. Uso del efecto de borde de la vegetación para la restauración ecológica del bosque tropical. México, Universidad Nacional Autónoma de México. Tip Revista Especializada en Ciencias Químico Biológicas. 2005; 8:91-98.

Rabeling C, Brown J, Verhaagh M. Newly discovered sister lineage sheds light on early ant evolution. 2008. The National Academy of Sciences of the USA.

Rivera L, Armbrecht I. Diversidad de tres gremios de hormigas en cafetales de sombra, de sol y bosque de Risaralda. Colombia- Meléndez, CO, Rev Colombiana Ent. 2005; 8.

Sarmiento CE. Metodologías de captura y estudio de las hormigas. 2003; 201-210 pp. En: Fernández, F (ed.), Introducción a las hormigas de la región neotropical. Bogotá, Instituto de Investigación de Recursos Biológicos Alexander von Humboldt.

Serna FJ, Vergara EV. Claves de identificación de subfamilias y géneros de hormigas de Antioquia y Choco Colombia. Chocó, Colombia. Graellsia. 2001; 1:37. 
Vaccaro CN, Mousques AJ. Hormigas cortadoras (géneros Atta y Acromyrmex) y tacurues en Entre Ríos. ConcordiaXII Jornadas Forestales de Entre Ríos. Instituto Nacional de Tecnologia Agropecuaria 1997; (34):7.

Vargas Rojas M. Introducción a la entomología general y agrícola. Santa Cruz, Universidad Santa Cruz de la Sierra, Universidad Autónoma Gabriel René Moreno, Instituto de Investigaciones Agrícolas el Vallecito. 1995; 9$266 \mathrm{pp}$.

Vergara Castrillón JC. Biologia, manejo y control de la hormiga arriera. Santiago de Cali, Gobernación Valle del Cauca. 2005; 20 pp.

Vergara Navaro EV, Echavaria Sanchez H, Serna Cardona F. Hormigas (Hymenoptera: Formicidae) asociadas al arboretum de la Universidad Nacional de Colombia. Boletin Sociedad Entomológico Aragonesano. 2007; 40:497-505.
Villareal H, Álvarez M, Córdoba F, Fagua G, Gast $\mathrm{F}$, Mendoza $\mathrm{H}$, et al. Manual de métodos para el desarrollo de inventarios de biodiversidad. Instituto de Investigación de Recursos Biológicos Alexander Von Humboldt, Bogotá, Colombia. 2006.

Ward, P.S. Subfamilia Pseudomyrmicinae. 2003; 331-333 pp. En: Fernández, F. (ed.), Introducción a las hormigas de la región neotropical. Bogotá, Instituto de Investigación de Recursos Biológicos Alexander von Humboldt.

Watkins JF. The identification and distribution of New World army ants (Dorylinae: Formicidae). Texas, Departamento de Biologia, Baylor University.1976; 168 pp. 